\title{
Crystallographic Attributes of a Shape-Memory Alloy
}

\author{
Division of Engineering \& Applied Science \\ California Institute of Technology \\ Pasadena, CA 91125
}

Shape-memory alloys are attractive for many potential applications. In an attempt to provide ideas and guidelines for the development of new shape-memory alloys, this paper reports on a series of investigations that examine the reasons in the crystallography that make (i) shape-memory alloys special amongst martensites and (ii) Nickel-Titanium special among shape-memory alloys.

\section{Introduction}

Shape-memory effect (SME) is the ability of certain metallic alloys to recover, on heating, apparently plastic deformation sustained below a critical temperature. Shape-memory alloys (SMAs) also display other related phenomena such as superor pseudo-elasticity. All these make SMAs very appealing for many potential applications. Though a sizable number of SMAs are known, applications have essentially been limited to nickeltitanium (at compositions close to equiatomic) due to a variety of reasons. The high cost of $\mathrm{Ni}-\mathrm{Ti}$, as well as the narrow temperature range in which it can be used, limit these applications. Therefore, it is important to improve known materials and develop new SMAs. This paper summarizes the results of a line of research motivated by these concerns. The goal is to understand if there are reasons in the crystallography that make

1. SMAs special among martensites and

2. Ni-Ti special amongst SMAs.

In doing so, it hopes to provide ideas and guidelines for the development of new SMAs.

The phenomenology of SME is well understood in a qualitative fashion, see for example Saburi and Nenno (1981) and Wayman (1992). The heart of this effect lies in the reversible or "thermoelastic" martensitic transformation that these crystalline solids undergo. A martensitic transformation is a temperature-induced first-order diffusionless phase transformation between the high temperature austenite phase and the low temperature martensite phase. In typical SMAs, the lattice of the austenite has higher symmetry than that of the martensite. This gives rise to more than one variant of martensite; variants are identical crystal lattices which are oriented differently with respect to the austenite.

Consider a specimen of a given shape in Fig. $1(a)$. It is in the austenite phase. Suppose momentarily that our specimen is a single crystal. On cooling, the austenite transforms to martensite. In particular, it transforms to a coherent fine-scale microstructure involving the different variants in such a manner that there is no macroscopic change in shape (Fig. 1(b)). This is known as self-accommodation. When loads are applied to the martensite, it deforms by converting one variant to another and forming a new coherent fine-scale microstructure (Fig. 1(c)). On heating, each variant transforms back to austenite. Since there is only one variant of austenite, all the strain is recovered and the specimen returns to its original shape (Fig. 1(a)), Notice that the strains are recoverable because the deformation below the transformation temperature is not due to slip, but rather due to the rearrangement of martensitic variants. Notice also that only certain strains are recovered: those that can be

Contributed by the Materials Division for publication in the JoURNAL of ENGINEERING Materials AND Technology. Manuscript received by the Materials Division February 11, 1998; revised manuscript received May 20, 1998. Guest Editors: H. Sehitoghu and Y. Chumlyakov. achieved by the rearrangement of martensitic variants. Larger strains introduce stress, giving rise to lattice defects and nonrecoverability.

If our specimen is a polycrystal, the situation is more complicated. At the high temperature it consists of a number of grains of austenite. As it is cooled, each grain transforms to a selfaccommodated microstructure of variants. As the polycrystal is deformed, each grain tries to accommodate the strain by adjusting its microstructure of martensitic variants. However, each grain is capable of sustaining a different class of microstructures due to the varying orientation. The recoverable strains in a polycrystal are the macroscopic averages of those inhomogeneous strain fields that may be accommodated in each grain by the rearrangement of martensite variants.

In summary, SME can only be observed in martensitic materials that are self-accommodating, that can form a large class of coherent microstructures and that can deform by changing microstructure at relatively small stresses. Our task is to find quantitatively any restrictions that these requirements impose on the crystallography of the material.

\section{Theory of Martensite Microstructure}

Ericksen (1980, 1984, 1986), James (1984), Ball and James $(1987,1992)$ and others have developed a theory in the framework of finite thermoelasticity to describe the behavior of martensitic materials. See Ball and James (1998) or Bhattacharya (1998) for detailed explanation. We assume that the behavior of the material is described by a (Helmholtz) free energy density which depends on temperature and deformation gradient. Above the transformation temperature, the free energy density has an absolute minimum at the austenite state; below the transformation temperature, it has an absolute minimum at the martensite state. The energy density satisfies all requirements of material frame-indifference and material symmetry.

Consider a single crystal of austenite at the transformation temperature. Choose this as the reference configuration and describe all other configurations of the crystal as deformations of this reference configuration. For example, the transformation may be described by the homogeneous deformation $y=U^{(1)} x$. $U^{(1)}$ is known as the transformation or the Bain matrix. However, due to the change in symmetry, there are $k$ variants of martensite with transformation matrices $U^{(1)}, U^{(2)}, \ldots, U^{(k)}$. We note that $k$ and the matrices $U^{(1)}, U^{(2)}, \ldots, U^{(k)}$ are known for any given material: they may be calculated from the change in symmetry and the change in lattice parameters during transformation. See Table 1 for a few important special cases (only $U^{(1)}$ is shown; $U^{(2)}, U^{(3)}, \ldots, U^{(k)}$ may be obtained from it by symmetry). Therefore, the identity matrix $I$ describes the austenite state while the matrices $U^{(1)}, U^{(2)}, \ldots, U^{(k)}$ describe the martensite state. Further, frame-indifference says that a rigid rotation does not change the energy of a crystal (or in other words, a rigid rotation does not change the state of the crystal). Therefore, we conclude that the deformation gradients corresponding to the 


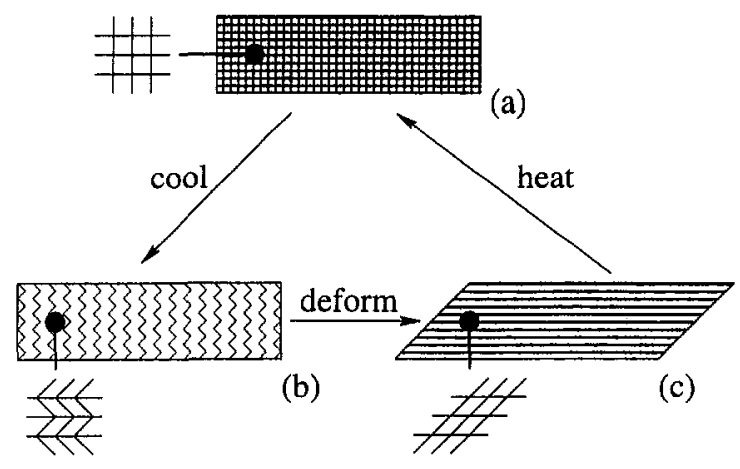

Fig. 1 The shape-memory effect

1. Austenite states are $R$ for any rotation matrix $R$;

2. Martensite states are $R U^{(1)}, R U^{(2)}, \ldots$, or $R U^{(k)}$ for any rotation matrix $R$.

Ball and James (1987) as well as Chipot and Kinderlehrer (1988) showed that energy minimization with such an energy density leads to fine-scale microstructure. Roughly, the idea is the following. When subjected to certain boundary conditions, the material tries to minimize its energy by making mixtures of the different variants of martensite while trying to satisfy all the coherence requirements. This leads to "minimizing sequences" which are interpreted as fine-scale microstructure. Ball and James (1987) also showed that the well-known phenomenological or crystallographic theory of martensite follows as a consequence of this theory. Since then, there has been much progress in understanding martensitic microstructure, and analytical tools like the "Young measure" and average compatibility conditions like the "minors relations" have been developed.

\section{The Wedge-Like Microstructure}

It is very common to observe a wedge-like or spear-like microstructure in SMAs. When the alloy is cooled from above the transformation temperature, wedge-shaped regions of martensite grow into the austenite. As shown in Fig. 2, the wedge consists of two sets of fine martensitic twins (fine alternating bands of two martensite variants) separated by a midrib. This microstructure provides an easy way for the initiation of transformation and is thus important for thermoelasticity and reversibility of transformation (Otsuka and Shimizu, 1969). To check whether a material can form a wedge, it is necessary to enforce two conditions: $(i)$ the deformation gradients within the wedge

Table 1 Some transformations and transformation matrices

\begin{tabular}{|c|c|c|}
\hline Transformation & $k$ & Transformation matrix \\
\hline $\begin{array}{l}\text { Cubic to tetragonal } \\
\text { Eg: Ni-Al }\end{array}$ & 3 & $\left(\begin{array}{lll}\alpha & 0 & 0 \\
0 & \alpha & 0 \\
0 & 0 & \beta\end{array}\right)$ \\
\hline $\begin{array}{l}\text { Cubic to orthorhombic } \\
\mathrm{Eg}: \gamma_{i}^{\prime} \mathrm{Cu}-\mathrm{Al}-\mathrm{Ni}\end{array}$ & 6 & $\left(\begin{array}{ccc}\alpha & \delta & 0 \\
\delta & \alpha & 0 \\
0 & 0 & \beta\end{array}\right)$ \\
\hline $\begin{array}{l}\text { Cubic to monoclinic-I } \\
\text { Eg: Ni-Ti }\end{array}$ & 12 & $\left(\begin{array}{ccc}\alpha & \delta & \epsilon \\
\delta & \alpha & \epsilon \\
\epsilon & \epsilon & \beta\end{array}\right)$ \\
\hline $\begin{array}{l}\text { Cubic to monoclinic-II } \\
\text { Eg: } \mathrm{Cu}-\mathrm{Zn}-\mathrm{Al}\end{array}$ & 12 & $\left(\begin{array}{lll}\alpha & \delta & 0 \\
\delta & \gamma & 0 \\
0 & 0 & \beta\end{array}\right)$ \\
\hline
\end{tabular}

The axis of monoclinic symmetry is $\langle 110\rangle_{\text {cubic }}\left(\langle 100\rangle_{\text {cubic }}\right)$ in monoclinicI (monoclinic-II).

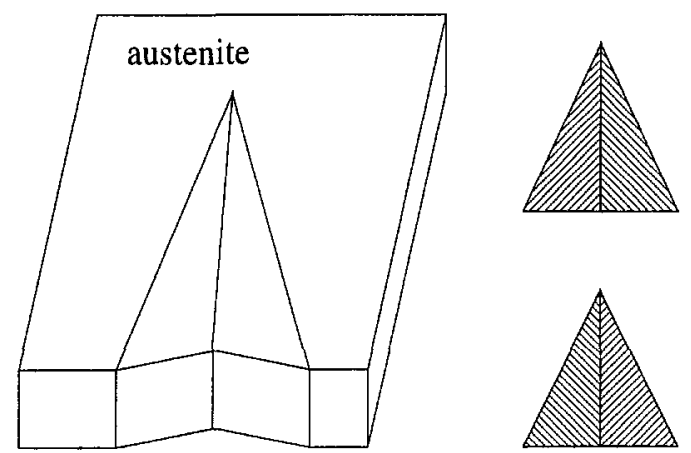

Fig. 2 Schematic view of a wedge-like microstructure

should correspond to martensite variants while the deformation gradients outside the wedge should correspond to the austenite and (ii) compatibility conditions have to be satisfied at the five interfaces - two twin interfaces, two austenite-martensite interfaces and one midrib.

Bhattacharya (1991) showed that the five compatibility conditions are too restrictive for any arbitrary material to satisfy. For example, a material that undergoes a cubic to tetragonal transformation can form a wedge if and only if the material parameters $\alpha$ and $\beta$ (cf. Table 1) satisfy the condition

$$
\alpha^{2}=\frac{\left(1-\beta^{2}\right)^{2}+4 \beta^{2}\left(1+\beta^{2}\right)}{\left(1-\beta^{2}\right)^{2}+8 \beta^{4}} .
$$

This describes a curve in $\alpha-\beta$ space (Fig. 3), and only those materials whose measured parameters lie on the curve can form a wedge. Similarly, in materials that undergo a cubic to orthorhombic transformation, the material can form a wedge if and only if the measured material parameters $\alpha, \beta$ and $\delta$ lie on a certain family of surfaces in $\alpha-\beta-\delta$ space. Furthermore, the theory predicts various geometrical features of the wedge.

Materials which are known to form a wedge satisfy these conditions very closely. We highlight $\mathrm{Ni}-\mathrm{Al}$ and $\mathrm{Cu}-\mathrm{Al}-\mathrm{Ni}$ in Table 2; also see Fig. 3. Further, the geometric details of the observed wedges agree very well with the predictions; for example the theory predicts that wedges with Type I twins in $\mathrm{Cu}-$ Al-Ni resemble the bottom-right of Fig. 2 while those with Type II twins the top-right in agreement with observations.

This calculation shows that microstructure often depends critically on the lattice parameters: a small change in lattice parameters can result in a significantly different class of microstructures and consequently significantly affect macroscopic properties. Therefore, it suggests that the ability of a material to display
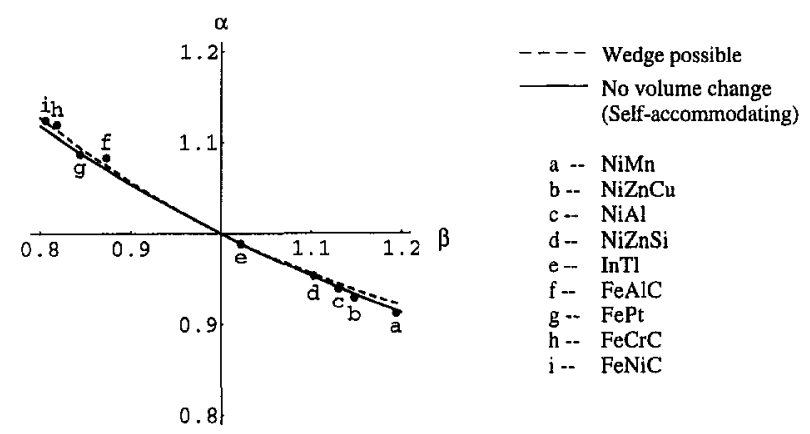

Fig. 3 The special relations on the transformation strain for wedge and self-accommodation in a cubic to tetragonal transformation. The measured lattice parameters of some alloys are also shown. Wedges have been observed in alloys $b, d, f$ and $i$ while alloys $a-e$ and $g$ are selfaccommodating (see Bhattacharya $(1991,1992)$ for details and references). 
Table 2 Wedge and self-accommodation: Comparison of theory and experiment. Wedges are seen in $\mathrm{Ni}-\mathrm{Al}$ and $\mathrm{Cu}$ Al-Ni; all three alloys are self-accommodating (see Bhattacharya $(1991,1992)$ for details and references)

\begin{tabular}{|c|c|c|c|}
\hline Material & $\begin{array}{c}\text { Observed } \\
\text { parameters }\end{array}$ & $\begin{array}{l}\text { Parameters } \\
\text { for a wedge }\end{array}$ & $\begin{array}{c}\text { Observed } \\
\text { volume change }\end{array}$ \\
\hline $\mathrm{Ni}-\mathrm{Al}$ & $\begin{array}{l}\alpha=0.9392 \\
\beta=1.1302\end{array}$ & $\begin{array}{l}\alpha=0.9445 \\
\beta=1.1302\end{array}$ & $0.305 \%$ \\
\hline $\mathrm{Cu}-\mathrm{Al}-\mathrm{Ni}$ & $\begin{array}{l}\alpha=1.0425 \\
\beta=0.9178 \\
\delta=0.0194\end{array}$ & $\begin{array}{l}\alpha=1.0425 \\
\beta=0.9193 \\
\delta=0.0194\end{array}$ & $0.297 \%$ \\
\hline $\mathrm{Ni}-\mathrm{Ti}$ & & & $0.023 \%$ \\
\hline
\end{tabular}

the SME may depend critically on the lattice parameters and consequently on composition.

\section{Self-Accommodation}

Self-accommodation is the ability of a SMA to transform from the austenite to the martensite with no macroscopic change in shape. Apart from being an inherent part of SME, Wayman (1992) and others have emphasized the role of self-accommodation as a necessary condition for SME.

Not every material that undergoes a martensitic transformation is self-accommodating. Consider, for example, a material where the volume of the martensite is smaller than that of the austenite. Clearly, the transformation leads to a change in volume (unless the material is subjected to large stresses) and the material is not self-accommodating. So, what are the conditions that guarantee that a material is self-accommodating?

From the arguments above, it is clear that volume preserving transformation is a necessary condition for self-accommodation. Bhattacharya (1992) showed that if the symmetry of the austenite is cubic, then this condition is also sufficient for self-accommodation. If on the other hand, the symmetry of the austenite is not cubic, the lattice parameters of the material have to satisfy additional restrictions which are extremely stringent and "nongeneric." Therefore, materials with cubic austenite have to satisfy a rather mild constraint, while materials with non-cubic austenite have to satisfy very restrictive non-generic conditions in order to be self-accommodating. It is highly unlikely that realistic materials will satisfy such nongeneric conditions. Consequently, only materials with cubic austenite which undergo almost volume preserving transformation are self-accommodating and hence shape-memory materials. This conclusion is in agreement with experimental observations as highlighted in Table 2 and Fig. 3; also see Bhattacharya (1992) for extensive comparison.

There is also a very interesting and probably important coincidence. Consider a cubic to tetragonal transformation. Volume preserving transformation corresponds to $\alpha^{2} \beta=1$ which describes a curve in $\alpha-\beta$ (transformation strain) space. As shown in Fig. 3, this curve is very close to the curve on which materials can form a wedge (see Eq. (2)) when $\alpha$ and $\beta$ are close to 1 , the range of experimental interest. Similarly, in a cubic to orthorhombic transformation, the volume preserving surface is close to the wedge forming surfaces in the $\alpha-\beta-\delta$ space. Thus, many materials with cubic austenite undergoing volume preserving transformations may also display a wedge!

Another interesting and important consequence of self-accommodation is that it is not possible to induce the two-way SME by making textured polycrystals.

Finally, a word about the theoretical methods. In the case of the wedge, we were analyzing a given microstructure. In contrast, here we are asking a broader question: is there any microstructure that is self-accommodating? Therefore, the method used to study the earlier problems--writing down and trying
Table 3 Summary of experimental observations of tensile recoverable strains (see Bhattacharya and Kohn (1996) for details and references)

\begin{tabular}{|c|c|c|}
\hline Material & $\begin{array}{l}\text { Single } \\
\text { crystal }\end{array}$ & Polycrystal \\
\hline $\mathrm{Ni}-\mathrm{Al}$ & $0-13 \%$ & Almost none \\
\hline $\mathrm{CuAlNi} / \mathrm{Cu}-\mathrm{Zn}-\mathrm{Al}$ & $2-9 \%$ & $\begin{array}{l}\text { Typically } \sim 2 \% \text {; up to } 6 \% \text { in special } \\
\text { ribbons }\end{array}$ \\
\hline $\mathrm{Ni}-\mathrm{Ti}$ & $3-10 \%$ & $5-8 \%$ in wires/sheets \\
\hline
\end{tabular}

to solve all the compatibility conditions - cannot be used here. No matter how many microstructures we check, there are always others. Therefore, we need methods to analyze general classes of microstructures. It is here that tools like the "Young measure" and average compatibility conditions like the "minors relations" are very useful.

\section{Geometrically Linear Theory of Martensite Micro- structure}

We now turn to calculating the recoverable strains that are inherent in the fundamental crystallography of a given material. This question turns out to be extremely difficult and at this time we are unable to carry through such a calculation in the geometrically nonlinear theory that we have been using so far.

However, it is possible to write a "geometrically linear" version of this theory by assuming infinitesimal displacements. This is similar to the theory used by Roitburd (1978) and Khachaturyan (1983) among others (see Bhattacharya (1993) for a detailed comparison). I believe that in the problems discussed below, this approximate theory gives reasonable results though the exact quantitative details may be different. In this geometrically linear theory, microstructures correspond to continuous displacements $u$ such that the infinitesimal strains $e=$ $\frac{1}{2}\left(\nabla u+\nabla u^{T}\right)$ "essentially" take values

1. 0 (Austenite states) or

$$
\text { 2. } e^{(1)}, e^{(2)}, \ldots, e^{(k)} \text { (Martensite states). }
$$

Here, $e^{(k)}=U^{(k)}-I$ are the transformation strains.

\section{Recoverable Strains}

Many alloys are known to be good SMAs as single crystals, but the extent to which they retain their SME in polycrystalline form is widely varied as highlighted by three alloys in Table 3 .

6.1 Single Crystal. We define the set of recoverable strains in a single crystal, $\delta$, as the set of all possible strains that can be achieved by mixing the different variants of martensite in

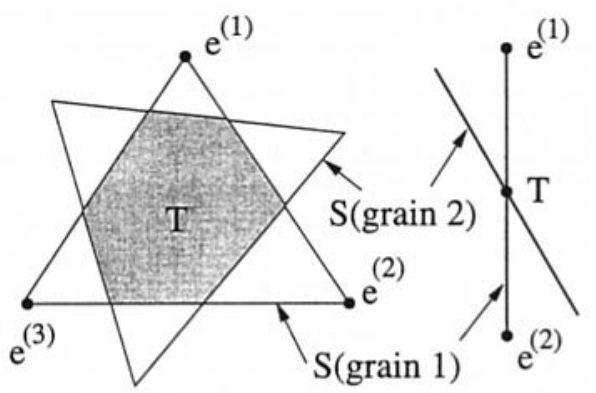

Fig. 4 The set of recoverable strains for a material undergoing large change in symmetry (left) and small change in symmetry (right). The recoverable strains $(S)$ in a single crystal is determined by the transformation strains of the variants while in a polycrystal it is determined by the interaction between the grains and is estimated by the set $T$. 
a coherent microstructure. We can calculate it for many interesting cases based on the following result of Bhattacharya (1993). If the transformation strains are pairwise compatible or twin related then $\delta$ is simply their convex hull, i.e., the set of all possible averages as shown in Fig. 4:

$$
S=\left\{e \| e=\sum_{i=1}^{k} \theta^{(i)} e^{(i)} \text { with } \theta^{(i)} \geq 0 \text { and } \sum_{i=1}^{k} \theta^{(i)}=1\right\} .
$$

This holds when the phase transformation is cubic to tetragonal, cubic to trigonal or cubic to orthorhombic, and Bhattacharya and Kohn (1996) have explicitly calculated this set. The cubic to monoclinic transformations are different, because some pairs of variants are not compatible. The exact sets are unknown but it is possible to obtain inner and outer estimates (Bhattacharya and Kohn, 1996 and Shu as Bhattacharya, 1998).

6.2 Polycrystal. A polycrystal is an assemblage of grains, each composed of the same material in a different orientation. The texture of a polycrystal (i.e., the shapes of the grains and their orientations ) can be represented by a rotation-valued function $R(x)$ which gives the orientation of the grain at $x$ relative to a reference single crystal.

A deformation of the polycrystal below the transformation strain is recoverable if it can be accommodated in each grain by the rearrangement of the martensite variants. The set of recoverable strain in a given grain, $\delta(x)$, is obtained by a suitable rotation of the set of recoverable strains of the reference single crystal: $\delta(x)=R(x) \delta R(x)^{T}$ (see Fig. 4). Therefore, if we assume that $(i)$ the microstructure size is much smaller than the grain size, which in turn is much smaller than the specimen size, and (ii) the grains are perfectly bonded, then the set of recoverable strains in a polycrystal, ${ }^{\circ}$, is the set of averages of strain fields that take values in $S(x)$ at each $x$. This set is rather difficult to calculate; however it is possible to obtain an easy inner bound or Taylor bound. Suppose a strain $e$ is recoverable in each grain, then it is certainly recoverable in the polycrystal. Therefore, $P \supset 5$ where

$$
\mathscr{J}=\cap_{x} \delta(x) .
$$

In other words, the polycrystal can recover all strains that are common to all the sets $\delta(x)$. The polycrystal can possibly recover larger strains by the cooperative effects between the grains - but, the Taylor bound is a surprisingly good estimate of the actual recoverable strains (Bhattacharya and Kohn, 1996 and 1997, Bhattacharya et al., 1997, Shu and Bhattacharya, 1998). Therefore we use the inner estimate to study recoverable strains.

Bhattacharya and Kohn (1996) found that the recoverable strains can depend critically on the change of symmetry during transformation. Briefly, polycrystals of materials which undergo small change of symmetry (cubic to tetragonal/trigonal) display no recoverable strain while polycrystals of materials which undergo large change of symmetry (cubic to orthorhombic/monoclinic) always display some change of symmetry. This result is in good agreement with experimental observations in Table 3: $\mathrm{Ni}-\mathrm{Al}$ undergoes cubic to tetragonal while $\mathrm{Cu}-\mathrm{Al}-\mathrm{Ni} / \mathrm{Cu}-\mathrm{Zn}$ $\mathrm{Al} / \mathrm{Ni}$ - $\mathrm{Ti}$ undergo cubic to orthorhombic/monoclinic transformation. The idea is explained in Fig. 4. A material undergoing small change of symmetry has few variants, so the set $\delta$ does not span deviatoric strain space (shown schematically as a line on the right). Therefore, if we rotate this set and intersect it according to (5), we find that the intersection reduces to a single point as shown on the right of Fig. 4. On the other hand, in materials undergoing a large change in symmetry we have a sufficient number of variants so that the set $s$ spans the five dimensional deviatoric strain space (shown schematically as a triangle on the left). Consequently, when we rotate it and intersect it according to (5) we obtain a non-trivial set $\Phi$ as shown on the left of Fig. 4.
Table 4 The predicted uniaxial recoverable extension for

\begin{tabular}{|c|c|c|c|c|c|c|}
\hline \multirow[b]{3}{*}{ Texture } & \multicolumn{6}{|c|}{ Recoverable strains (\%) } \\
\hline & \multicolumn{3}{|c|}{ TiNi } & \multicolumn{3}{|c|}{$\mathrm{CuZnAl}$} \\
\hline & $\epsilon_{R}^{i}$ & $\epsilon_{R}^{e}$ & $\epsilon_{R}^{i j}$ & $\epsilon_{R}^{i}$ & $\epsilon_{R}^{e}$ & $\epsilon_{R}^{i f}$ \\
\hline random & 2.3 & 2.3 & 2.3 & 1.3 & 1.7 & 1.7 \\
\hline$\langle 110\rangle$ wire/rod & 6.0 & 7.5 & - & 1.3 & 1.7 & - \\
\hline$\langle 111\rangle$ wire/rod & 3.9 & 9.6 & - & 1.7 & 1.7 & - \\
\hline$\{100\}$ film/ribbon & 2.3 & 2.3 & 2.3 & 4.6 & 7.1 & 7.1 \\
\hline$\{110\}$ film & 2.3 & 2.3 & 2.3 & 1.5 & 1.7 & 1.7 \\
\hline$\{111\}$ film/sheet & 4.6 & 5.3 & 8.1 & 1.4 & 1.9 & 5.9 \\
\hline
\end{tabular}
various textures

While the effect of symmetry explains much experimental observation, it fails to make any distinction within alloys which undergo a large change in symmetry. In particular, it is unable to explain why $\mathrm{TiNi}$ is so much better than $\mathrm{Cu}$-based SMAs since both undergo cubic-monoclinic transformation and have similar transformation strains. Shu and Bhattacharya (1998) have systematically explored the role of texture to understand this difference. They develop an inner bound $\epsilon_{R}^{i}$ and inner estimate $\epsilon_{R}^{e}$ for the recoverable strain for a polycrystal with given texture. Table 4 compares the predicted recoverable strain in $\mathrm{TiNi}$ and $\mathrm{CuZnAl}$ for various textures. Notice that for specimens with random texture, the recoverable strain in either alloy is predicted to be rather small. Wires and rods made of these materials have either a $\langle 110\rangle$ or a $\langle 111\rangle$ texture: notice that in either of these cases the strains are much larger in TiNi compared to CuZnAl. Similarly, common rolling textures are also good textures for large recoverable strains in $\mathrm{TiNi}$, but not in CuZnAl. All these predictions are in good agreement with experimental observation.

We conclude by commenting on some recent efforts to produce SMA thin-films by sputtering. It is possible to derive an inner bound for the maximum recoverable strains in very thin films $\epsilon_{R}^{i f}$ (Shu and Bhattacharya, 1998). Sputtering TiNi produces films with either random or $\{110\}$-film texture. Table 4 shows that both these textures yield only very small strains, in agreement with the limited strains observed in films. Finally, large strains are expected in TiNi films with $\{111\}$ texture and $\mathrm{CuZnAl}$ films with $\{100\}$ texture.

\section{Conclusions}

These results provide the following broad principles.

- The microstructure of a martensitic material, and consequently its macroscopic properties, depend critically on the lattice parameters and consequently on composition.

- Only materials with cubic austenite that undergo a volume preserving transformation can be shape-memory materials.

- It is not possible to induce the two-way shape-memory effect by making specially textured polycrystals.

- Materials with monoclinic martensite will display the best shape-memory effect in polycrystalline form.

- Polycrystals of materials with tetragonal (or trigonal) martensite will display the shape-memory effect only in applications where the required recovery is limited to uniaxial tension or compression. Even in such applications it is necessary to process the material, perhaps by rolling, drawing or by repeated deformation, followed by annealing, to endow it with $\langle 100\rangle$ texture (or $\langle 111\rangle$ texture). Despite all this, it is natural to expect some unrecoverable strains.

- Ni-Ti is such an outstanding SMA as wires and sheets because it undergoes a large change of symmetry during transformation and the texture that develops during roll- 
ing, extrusion and drawing is extremely favorable from the point of view of large recoverable strains.

- The random or $\{110\}$ sputtering texture is not a good thin-film texture for $\mathrm{Ni}-\mathrm{Ti}$ or $\mathrm{Cu}-\mathrm{Zn}-\mathrm{Al}$. Large strains are expected in TiNi films with $\{111\}$ texture and $\mathrm{CuZnAl}$ films with $\{100\}$ texture.

\section{Acknowledgments}

This research started when I was a graduate student at the University of Minnesota and continued through my post-doctoral stay at the Courant Institute. I am deeply indebted to Prof. Richard D. James for introducing me to this subject and for continuing to inspire me, and Prof. Robert V. Kohn for his continuing support and encouragement. I gratefully acknowledge financial support from AFOSR (at Minnesota and Caltech), ARO (at Courant), NSF (at Minnesota, Courant and Caltech), and ONR (at Caltech).

\section{References}

Ball, J. M., and James, R. D., 1987, "Fine Phase Mixtures as Minimizers of Energy," Archive for Rational Mechanics and Analysis, Vol. 100, pp. 13-52.

Ball, J. M., and James, R. D., 1992, "Proposed Experimental Tests of a Theory of Fine Microstructure," Philosophical Transactions of the Royal Society of London, Vol. 338A, pp. 389-450.

Ball, J. M., and James, R. D., 1996, Fine Microstructures, in preparation.

Bhattacharya, K., 1991, "Wedge-Like Microstructure in Martensite," Acta Metallurgica et Materialia, Vol. 39, pp. 2431-2444.

Bhattacharya, K., 1992, "Self-Accommodation in Martensites," Archive for Rational Mechanics and Analysis, Vol. 120, pp. 201-244.

Bhattacharya, K., 1993, "Comparison of the Geometrically Nonlinear and Linear Theories of Martensitic Transformation," Continuum Mechanics and Thermodynamics, Vol. 5, pp. 205-242.
Bhattacharya, K., 1998, "A Theory of Martensitic Microstructure and its Implications for the Shape-Memory Effect," to appear in Shape-Memory Effect, ed. Airoldi, G., Miyazaki, S. and Muller, I, eds., Trans. Tech. Pub.

Bhattacharya, K., and Kohn, R. V., 1996, "Symmetry, Texture, and the Recoverable Strain of Shape-Memory Polycrystals," Acta Materialia, Vol. 44, pp. 529542 .

Bhattacharya, K, and Kohn, R. V., 1997, "Elastic Energy Minimization and the Recoverable Strains of Polycrystalline Shape-Memory Materials," Archive for Rational Mechanics and Analysis, Vol. 139, pp. 99-180.

Bhattacharya, K., Kohn, R. V., and Shu, Y. C., 1997, "The Taylor Estimate of Recoverable Strain in Shape-Memory Polycrystals," The Proceedings of the IUTAM Symposium on Transformation Problems in Composite and Active Materials, Bahei-el-Din, Y. A. and Dvorak, G. J., eds., Kluwer, in press.

Chipot, M., and Kinderlehrer, D., 1988, "Equilibrium Configurations of Crystals," Archive for Rational Mechanics and Analysis, Vol. 103, 237-277.

Ericksen, J. L., 1980, "Some Phase Transitions in Crystals," Archive for Rational Mechanics and Analysis, Vol. 73, pp. 99-124.

Ericksen, J. L., 1984, "The Cauchy and Born hypothesis for crystals," Phase Transformations and Material Instabilities in Solids, Gurtin, M. E., ed., Academic Press, pp. 61-78.

Ericksen, J. L., 1986, "Stable Equilibrium Configurations of Elastic Crystals," Archive for Rational Mechanics and Analysis, Vol. 94, pp. 1-14.

James, R. D. 1986, "Displacive Phase Transformations in Solids," Journal of Mechanics and Physics of Solids, Vol, 34, pp. 359-394.

Khachaturyan, A. G., 1983, Theory of Structural Transformations in Solids, Wiley, New York.

Otsuka, K., and Shimizu, K., 1969, "Morphology and Crystallography of Thermoelastic $\gamma$ Cu-Al-Ni Martensite," Japanese Journal of Applied Physics, Vol. 8, pp. 1196-1204.

Roitburd, A., 1978, "Martensitic Transformation as a Typical Phase Transformation in Solids," Solid State Physics, Vol. 33, pp. 317-390.

Saburi, T., and Nenno, S., 1981, "The Shape Memory Effect and Related Phenomena," Proc. International Conference on Solid-Solid Phase Transformations, H. I. Aaronson et al., eds., The Metallurgical Society AIME, New York, pp. $1455-1479$.

Shu, Y.C., and Bhattacharya, K., 1998, "The Influence of Texture on the Shape-Memory Effect in Polycrystals," Acta Materialia, in press.

Wayman, C., 1992, "Shape Memory and Related Phenomena," Progress in Materials Science, Vol. 36, pp. 203-224. 